\title{
1 Plasticity in Ligand Recognition at Somatostatin Receptors
}

3 Michael J. Robertson ${ }^{1,2, *}$, Justin G. Meyerowitz ${ }^{1,2,3,{ }^{*},}$ Ouliana Panova ${ }^{1,2}$, Kenneth

4 Borrelli ${ }^{4}$, Georgios Skiniotis ${ }^{1,2}$

$6{ }^{1}$ Department of Molecular and Cellular Physiology, Stanford University School of 7 Medicine, Stanford, CA, USA

$8{ }^{2}$ Department of Structural Biology, Stanford University School of Medicine, Stanford, CA, 9 USA

10 '3epartment of Anesthesiology, Perioperative and Pain Medicine, Stanford University

11 School of Medicine, Stanford, CA 94305, USA.

12 4Schrödinger, New York, NY 10036, USA.

13 *These authors contributed equally.

14 Correspondence: yiorgo@stanford.edu

16 Abstract

17 Somatostatin is a signaling peptide that plays a pivotal and wide-ranging role in 18 physiologic processes relating to metabolism and growth through its actions at 19 somatostatin receptors (SSTRs). Members of the somatostatin receptor subfamily, 20 particularly SSTR2, are key drug targets for neuroendocrine neoplasms, with several

21 synthetic peptide agonists currently in clinical use. Here, we show the cryogenic electron

22 microscopy (cryo-EM) structures of active-state SSTR2 in complex with heterotrimeric Gi

23 protein and either the endogenous ligand SST14 or the FDA-approved drug octreotide. 
24 Complemented by biochemical assays and molecular dynamics simulations, these

25 structures reveal key details of ligand recognition, receptor activation, and subtype-

26 selectivity at somatostatin receptors. We find that SSTR ligand recognition is highly

27 diverse, as demonstrated by ligand-induced conformational changes in ECL2, substantial

28 sequence divergence across subtypes in extracellular regions, and loss of ligand binding

29 upon several structurally homologous substitutions between subtypes. Despite this

30 complexity, we rationalize several previously described sources of SSTR subtype

31 selectivity and identify an additional key interaction for SSTR2/3/5 specific binding. These

32 results shed light on the basis of ligand recognition by somatostatin receptors and provide

33 valuable insights for structure-based drug discovery at these targets.

\section{Introduction}

Somatostatin (SST) is a 14- (SST14) or 28-residue (SST28) peptide that regulates

37 crucial aspects of animal physiology by suppressing metabolic- and growth-related

neuroendocrine signaling systems including insulin/glucagon, thyroid-stimulating

39 hormone, prolactin, growth hormone, and digestion-related processes ${ }^{1}$. These actions of

40 somatostatin are mediated by somatostatin receptors (SSTRs), which are G protein-

41 coupled receptors (GPCRs) that signal through the inhibitory Gi/o family of G proteins ${ }^{2}$ to

42 prevent the release of secretory hormone vesicles. Neuroendocrine tumors (NETs)

43 generally overexpress SSTRs, allowing somatostatin agonists to function as diagnostic

44 imaging tracers ${ }^{3}$ and therapeutic agents for hormone excess disorders caused by NETs,

45 including carcinoid syndrome, gigantism, acromegaly, hyperthyroidism, and Cushing's $46 \quad$ disease $^{4}$. 
The SSTR subfamily includes five distinct isoforms, SSTR1-5, with SSTR2 having two C-terminal splice variants, SSTR2a and SSTR2b. These receptors have varied tissue expression profiles, including differential expression across NETs. SSTR2 is highly overexpressed in most NETs, and thus this subtype is the primary target for clinical

52 agonists. All FDA-approved somatostatin agonists currently in use are peptide analogs of 53 SST14, including the SSTR2-selective drugs octreotide and lanreotide ${ }^{5}$, and the newer

54 SSTR2-, 3-, and 5-selective pasireotide ${ }^{6}$. Abundant evidence suggests that distinct SSTR

55 isoforms play important roles in a variety of tumor subtypes beyond NETs ${ }^{1}$, elevating the

56 need to probe isoform-specific function with more selective pharmacologic tools ${ }^{7}$.

57 Furthermore, the development of orally bioavailable small-molecule SSTR agonists could substantially improve treatment of NETs, as these agonists are often required for chronic

59 therapy ${ }^{8}$. However, the lack of structural information for either the inactive or active state

60 of any of the SSTR subtypes, and the lack of a structural framework for SSTR ligand

61 binding and selectivity, is a substantial impediment for further drug discovery.

All SSTR subtypes exhibit nearly identical affinity for the endogenous peptide

64 SST14, despite having only $40-55 \%$ sequence homology and substantial variation in the extracellular region encompassing the ligand interaction site. Radioligand binding assays

66 have suggested that both the extracellular half of the transmembrane (TM) domains and

67 the extracellular loops (ECLs) play a role in ligand binding and subtype selectivity ${ }^{9}$.

68 However, which precise residue(s) are critical depends upon both subtype and ligand, 69 suggesting a complex interaction landscape between SSTRs and their ligands. Here, we 
70 used cryo-EM, biochemical assays, and atomistic simulations to obtain structural and

71 mechanistic insights into ligand recognition and selectivity at somatostatin receptors. The

72 results highlight surprising heterogeneity and diversity of ligand-protein recognition in a

73 subfamily of receptors that are activated by the same endogenous ligand.

\section{SSTR2 Couples Robustly to Gi/o Proteins}

To better understand the signaling landscape of SSTR2 and identify the optimal G

77 protein partner for forming stable receptor-G protein complexes for structural studies we

78 employed bioluminescence resonance energy transfer (BRET)-based activation assays

79 to screen for coupling by the majority of $G$ proteins ${ }^{10}$ (Extended Data Fig. 1a, b).

80 Consistent with previous studies, SSTR2 interacted robustly with all Gi/o family G

81 proteins. Although Gi1 has traditionally been the preferred Gi/o type for structural studies,

82 our results identified it as the weakest SSTR2 signaling partner within the Gi/o family.

83 Interestingly, we also observed ligand-induced interaction with G15, which belongs to the

84 Gq family, and G12, which forms a distinct G protein subfamily with G13, although in both

85 cases with weak potency compared to Gi/o activation. No other G proteins tested of the

$86 \mathrm{Gq}, \mathrm{Gs}$, or G12/13 families were activated by SSTR2. As prior studies have established

87 Gi3 as a likely physiologic coupling partner, we used this G protein for complexation and

88 further studies. To resolve receptor stability issues at low ionic strength during purification,

89 we swapped the third intracellular loop (ICL3) and the bottom 9 residues of

90 transmembrane helix 6 (TM6) for that of the kappa opioid receptor (KOR) ${ }^{11}$. This strategy

91 also enabled us to employ a nanobody recognizing the KOR ICL3 in order to determine

92 the inactive-state cryo-EM structure of SSTR2 reported in a companion manuscript 
93 (Robertson et al. ${ }^{12}$ ). This construct, denoted SSTR2 «ICL3, coupled to Gai3

94 indistinguishably from wild-type receptor in BRET assays (Extended Data Fig. 1c). Thus,

95 we used SSTR2 ${ }_{\kappa I C L 3}$ for complexation with a dominant-negative G $\alpha і 3 \beta \gamma$ heterotrimer and

96 scFv16, an antibody fragment that aids complex stability and has been employed in

97 numerous cryo-EM studies ${ }^{13,14}$. High-quality complex preparations were obtained with

98 both octreotide and SST14, and cryo-EM enabled us to determine maps with global

99 resolutions of $2.9 \AA$ and $2.5 \AA$, respectively (Fig. 1a, Extended Data Fig. 2, 3). The two

100 structures show near-identical coupling between the receptor and Gai3, in a way that is

101 similar to the receptor-G protein coupling interaction of the $\mu$-opioid receptor in complex

102 with Goi115 (Extended Data Fig. 4a). At the resolutions obtained, water-mediated

103 interactions can be resolved between the $\alpha 5$ helix of Gai3 and the intracellular cavity of

104 the receptor, bridging the TM3 DRY motif D1393.49 (Ballesteros-Weinstein notation ${ }^{16}$ ),

$105 \mathrm{R} 140^{3.50}, \mathrm{~T} 78^{2.39}, \mathrm{R} 155^{4.38}, \mathrm{~S} 150$ and the backbone carbonyl of P147 on the receptor with

106 E350 N347 and the backbone carbonyl of C351 on the G protein (Extended Data Fig. 4b,

107 c). These density interpretations were further confirmed by JAWS simulations ${ }^{17}$, which

108 probe occupancies and absolute binding free energies of water molecules, that 109 recapitulated the experimentally observed water positions at the receptor-G protein 110 interface (Extended Data Fig. 4b, c).

111

112 Agonist-Bound SSTR2 Structures Reveal Flexible Ligand Accommodation

113 Both SST14 and octreotide were well-resolved in the orthosteric binding site, with

114 the conserved Phe-L/DTrp-Lys-Thr motif, common to most SSTR-binding peptides,

115 located in the core of the receptor (Fig 1b, c, e). Comparing the ligand-bound structures 
116 to our apo, inactive-state structure (Fig. 1d), the SST14/octreotide tryptophan buries into

117 a hydrophobic pocket, breaking a hydrogen bond between Q126 3.36 and $\mathrm{Y} 273^{6.52}$ that is

118 present in the apo structure (Fig. 1e). The lysine (L5 of octreotide and L9 of SST14) forms

119 a salt bridge with $\mathrm{D} 122^{3.32}$, causing this aspartate and $\mathrm{Y} 273^{6.52}$ to shift position, which in

120 turn drives $\mathrm{F} 92^{2.53}$ to shift down compared to the inactive state, pushing against TM3. The

121 combination of the steric pressure from F92 ${ }^{2.53}$ and the loss of the Q126.36-Y273 ${ }^{6.52}$

122 hydrogen bond allows TM3 to be displaced away from TM6 by roughly $3.5 \AA$. This

123 movement creates the necessary space for the PIF-motif phenylalanine (F267 6.46$)^{18}$ to

124 alter position and $\mathrm{Y} 312^{7.53}$ of the NPxxY motif ${ }^{19}$ to engage in TM6 opening and activation

125 of the receptor (Fig. 1f). The Q126 $3.36-Y 273^{6.52}$ TM3/TM6 hydrogen bond motif is

126 somewhat unique, with only SSTR2, SSTR3, SSTR5, and the melanin-concentrating

127 hormone receptors 1 and $2(\mathrm{MCH} 1 / 2)$ having a $\mathrm{Q}^{3.36}$, and only SSTR2, SSTR3, and

$128 \mathrm{MCH} 1$ possessing $\mathrm{Q}^{3.36}$ paired with $\mathrm{Y}^{6.52}$. Of note, $\mathrm{W} 269^{6.48}$, often cited as a 'toggle switch'

129 residue that changes conformation upon activation of several family A GPCR ${ }^{20}$, is largely

130 unchanged between the inactive and active-state SSTR2 conformations (Fig. 1f).

131 While the receptor activation mechanism appears the same between the two

132 ligands, the extracellular receptor loops exhibit strikingly different behavior in the two

133 structures. In the case of the 8-residue, SSTR2-selective octreotide, ECL2 folds down

134 from its position in the apo state to form a cover over the ligand, making hydrogen bonds

135 with the peptide and providing a cap involving W188 (Fig. 2a). ECL3 moves inward from

136 the apo state and covers the sidechain of the N-terminal D-phenylalanine of octreotide

137 with P286 (Extended Data Fig. 5a), providing hydrophobic packing. By contrast, the

138 substantially larger SST14 peptide pushes ECL2 to be slightly more extended than in the 


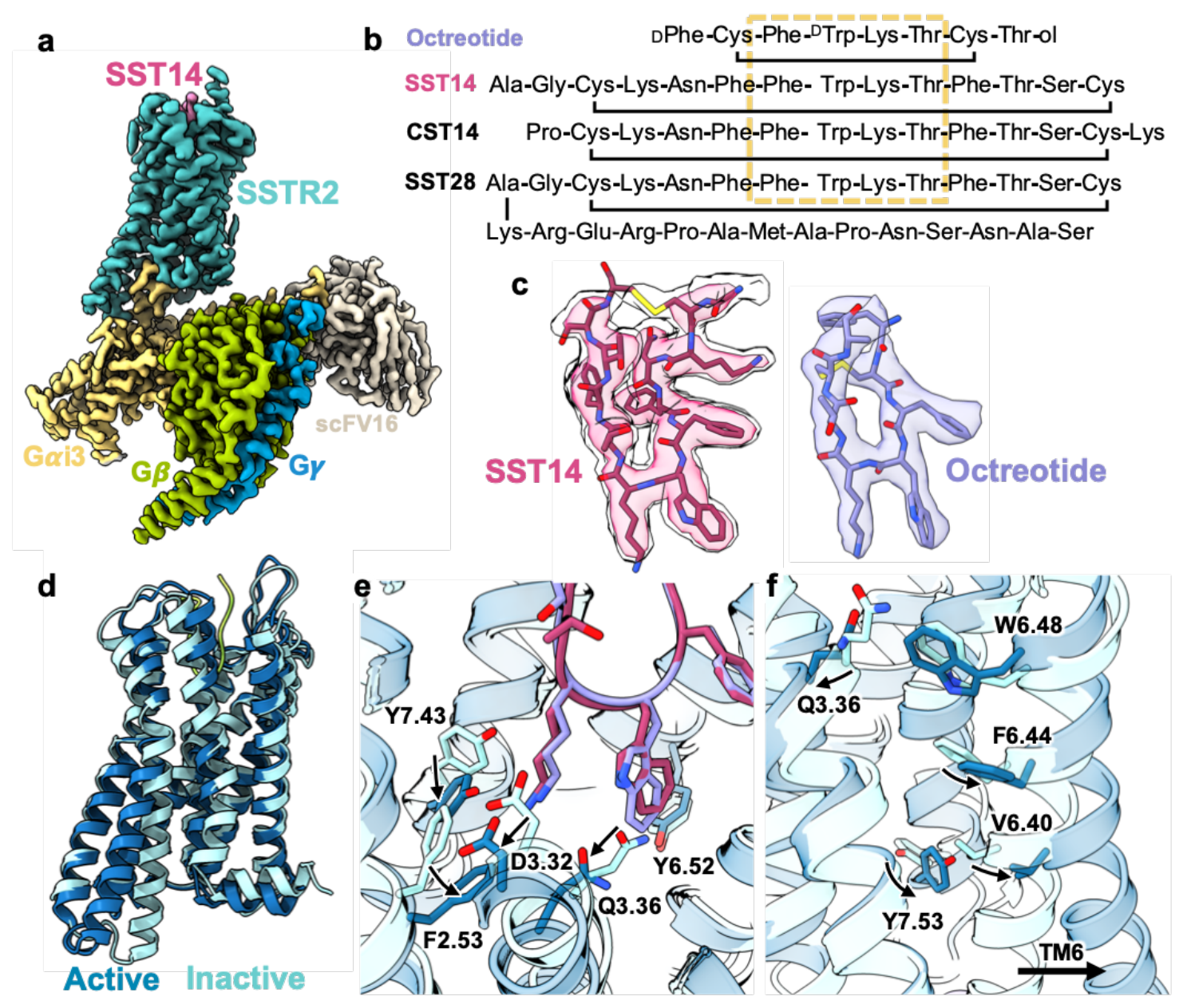

Figure 1. Cryo-EM structures of SST14 or octreotide activated SSTR2-Gi protein complex

a, Active-state SSTR2 (teal) bound to SST14 (magenta) in complex with G $\alpha$ i3 (goldenrod), G $\beta$ (lime), G $\gamma$ (cerulean), and scFv16 (bone). b, Peptide agonists of SSTR2, with conserved key binding region boxed in goldenrod. c, map-model fit of SST14 (magenta, left) at two different threshold levels and octreotide (lavender, right). d, Alignment of active (blue) and inactive (arctic blue) SSTR2 receptors. e, Peptide-induced conformational changes in the extracellular cavity during activation in response to SST14 (magenta) or octreotide (lavender). f, Conformational changes on the intracellular site of SSTR2 upon ligand binding and G protein coupling.

140 (Fig. 2b). ECL3 is pulled inward towards the ligand in a similar overall fashion between

141 SST14 and octreotide, although the P286 hydrophobic cap is in closer proximity with

142 octreotide (Extended Data Fig. 5d). To probe the behavior of ECL2 we performed 
143 molecular dynamics (MD) simulations in triplicate at $1 \mu$ s timescale. The MD trajectories

144 of apo-SSTR2 demonstrate that ECL2 remains in an upward state and does not

145 spontaneously fold over the extracellular cavity (Fig. 2c), consistent with the cryo-EM

146 structure. However, when simulations were performed starting from the apo state with

147 octreotide added in the vicinity of the binding pocket, rapid repositioning of ECL2 was

148 observed, demonstrating that this is a ligand-induced conformation of the receptor.

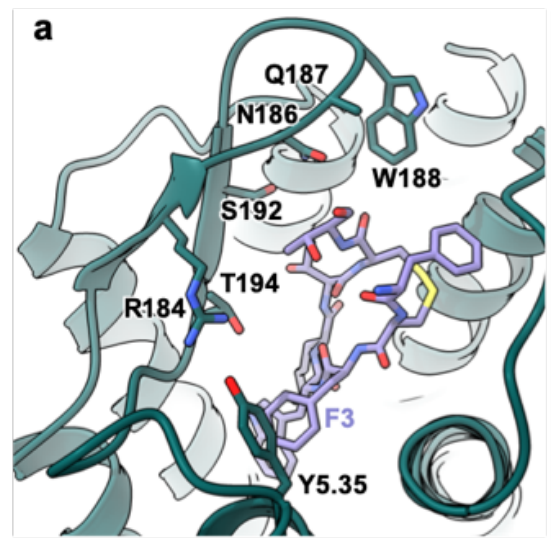

b

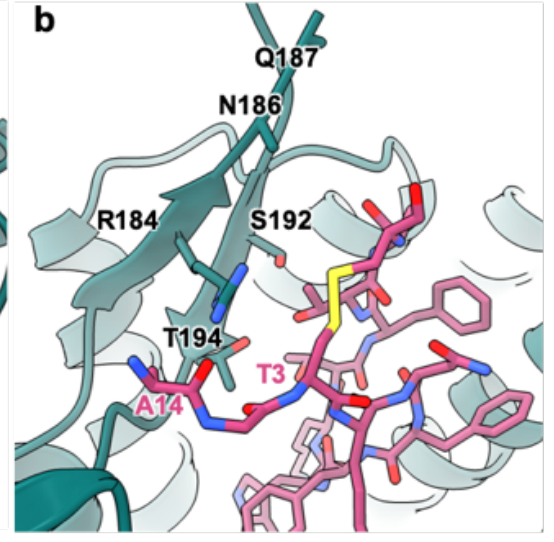

c Octreotide $\longleftrightarrow \mathrm{SST} 14$

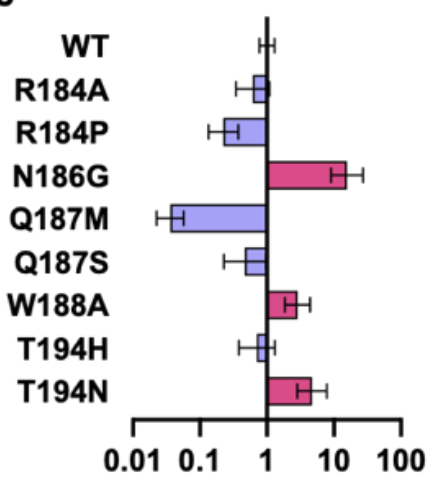

d $邓$

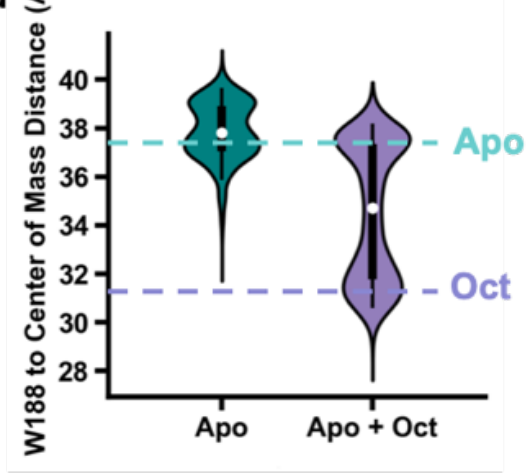

Figure 2. Differential SSTR2 ECL2 interactions with octreotide and SST14.

a, ECL2 interactions of SST14 (magenta)-bound SSTR2 (teal). b, ECL2 interactions of octreotide (lavender)-bound SSTR2 (teal). c, Fold change in selectivity in favor of octreotide (lavender) or SST14 (magenta) as compared to selectivity of wild-type SSTR2. Error bars are $95 \% \mathrm{Cl}$. d, Violin plots of the distance between W188 and the center of mass of the receptor during MD simulations of either apo inactive SSTR2 (teal) or apo SSTR2 with octreotide introduced (lavender). 

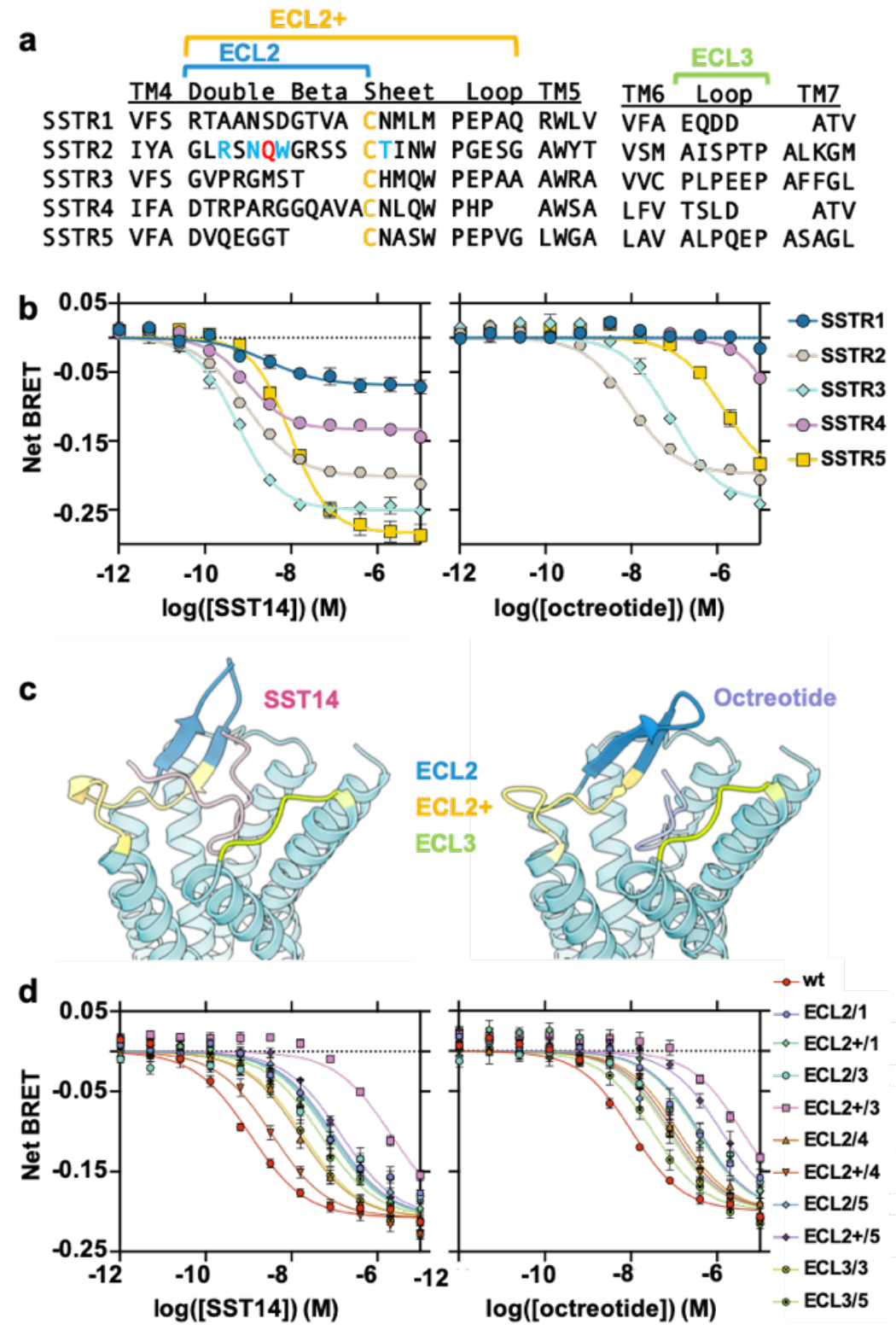

Figure 3. Role of ECL2 and ECL3 in SSTR subtype selectivity.

a, Alignment of ECL2 and ECL3 of SSTRs, with swapped regions denoted with brackets. b, Dose-response curves of SSTR isoform-dependent activation of $\mathrm{Gi} 3$ BRET biosensor in response to SST14 (left) or octreotide (right). c, Structure of SST14 bound SSTR2 (left) and octreotide bound SSTR2 (right) with swapped regions colored. d, Dose-response curves of SSTR extracellular loop (ECL) swap-dependent activation of Gi3 BRET biosensor in response to SST14 (left) or octreotide (right). Error bars are S.E.M..

154 (Fig. 2d, Extended Data Fig. 5 b, c). R184 is positioned to potentially form a hydrogen

155 bond with the backbone of the SST14 N-terminus (Fig. 2b). In contrast, in the octreotide-

156 bound receptor, R184 forms an intra-receptor hydrogen bond with Y205 ${ }^{5.35}$ (Fig. 2a),

157 holding this residue in place to form an edge-face pi-pi interaction with F3 of octreotide.

158 Mutation of R184 to either alanine or proline (analogous to SSTR1 and SSTR3, 159 respectively) indeed substantially reduces SSTR2 activation by both compounds 
160 (Extended Data Fig. 5b, c). We also replaced T194 with either histidine or asparagine,

161 present in SSTR3 and SSTR1, 4, \& 5, respectively, which would disrupt both polar

162 contacts made by T194 and provide a steric clash in the case of histidine. We found that

163 both T194H and T194N impact the Gi3 signaling of both compounds (Extended Data Fig.

$1645 \mathrm{~b}, \mathrm{c}) . \mathrm{N} 186$ is observed near octreotide and positioned to make an indirect polar

165 interaction with the ligand, while this residue is not resolved in the SST14 structure,

166 presumably due to its variable positioning further up in the raised ECL2. Notably, mutation

167 of N186 to glycine results in increased selectivity for SST14 over octreotide (Fig. 2d). On

168 the other hand, Q187 at the tip of ECL2 does not appear to interact with either ligand

169 directly, and the Q187S mutant retains identical Gi3 response and ligand selectivity

170 compared to wild-type (Extended Data Fig. 5b, c). Curiously, the Q187M mutation

171 enhances octreotide signaling (Fig. 2d), perhaps by generating additional hydrophobic

172 packing with the neighboring W188. Finally, W188 occurs in an unresolved region in the

173 SST14 structure but is resolved packed against octreotide's C-terminal threonine-ol.

174 Consistent with the notion that W188 participates only in octreotide binding, the W188G

175 mutant altered selectivity to be unfavorable to octreotide (Fig. 2d).

177 Subtype Selectivity at SSTRs is Multifaceted

178 The demonstration of ECL-driven ligand selectivity in SSTR2 raises questions

179 about how the ECL2 and ECL3 of other SSTR subtypes contribute to ligand coordination

180 and selectivity, as their ECL regions are highly divergent in length and sequence

181 compared to SSTR2 (Fig. 3a). All SSTRs have comparable response to SST14 in BRET

182 assays; however, octreotide activates SSTR2 most potently, with a reduced response at 
183 SSTR3 and SSTR5, and almost no activity at SSTR1 and SSTR4, which we recapitulated

184 in our functional assays (Fig. 3b). To probe the role of individual SSTR ECLs on ligand

185 recognition, we generated chimeric constructs of SSTR2 and used BRET-based assays

186 to monitor Gi3 activation in response to SST14 and octreotide. In these chimeras we

187 swapped the SSTR2 ECL2 or ECL3 for the corresponding sequence of other SSTR

188 subtypes (Fig. 3a, c). For ECL2 we swapped the sequence either through the conserved

189 cysteine (denoted "ECL2/X") or in its entirety (denoted "ECL2+/X", with X being the

190 isoform number of origin). Surprisingly, nearly all ECL swaps impaired ligand recognition

191 of SST14 and octreotide to some degree, with generally far greater impairment for SST14

192 than octreotide (Fig. 3d). Only the swap of the entirety of ECL2 from SSTR4 (ECL2+/4)

193 selected against octreotide, and in this case, improved SST14-induced signaling

194 compared to the shorter SSTR4 ECL2 swap. Further, exchanging ECL3 with the shorter

195 ECL3/TM7 of SSTR1 and SSTR4 completely abolished G protein activation in response

196 to either ligand.

197 The complexity of SST14 recognition can be further demonstrated through point

198 mutations. H192 of SSTR3 is in a homologous position to T194 in SSTR2, which is

199 located immediately next to the conserved ECL2 cysteine (C193 in SSTR2) that forms a

200 disulfide bond with the conserved $C^{3.25}$ (C115.25 in SSTR2). Consistent with the

201 observation that T194 in SSTR2 is critical for ligand recognition, we observe that H192T

202 and H192A in SSTR3 are both deleterious to SST14-stimulated G protein activation

203 (Extended Data Fig. 5f). Thus, despite the ECL2/C 3.25 disulfide bond anchoring the

204 position of this residue in space with respect to TM3 in all isoforms, mutation of the

205 adjacent ECL2 T194 in SSTR2 or H192 in SSTR3 is functionally deleterious. Taken 

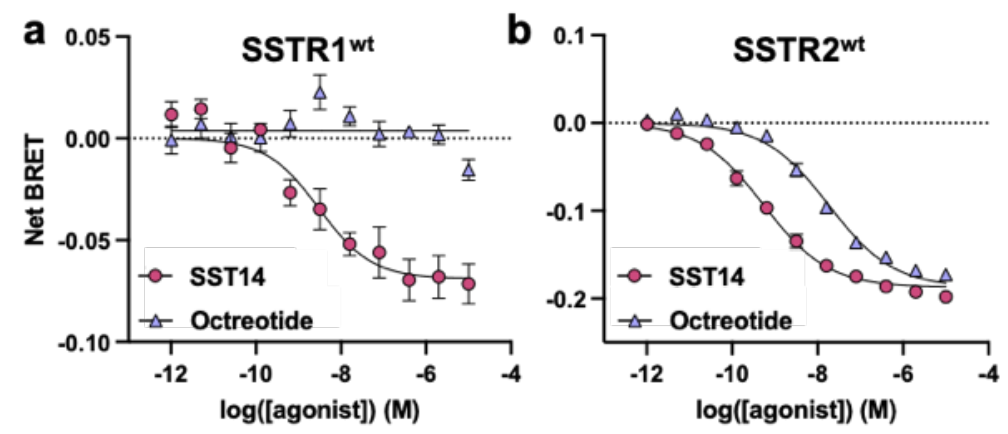

Figure 4. Subtype-selective point mutations in SSTR1 and SSTR2. Doseresponse curves for a, SSTR1- and $\mathbf{b}$, SSTR2-dependent activation of $\mathrm{Gi} 3$ in response to SST14 and octreotide. c, Dose-response curves for SSTR2 ${ }^{\mathrm{N} 276 \mathrm{Q}}$ dependent activation of Gi3, d, Overlay of SSTR2 (teal) bound to SST14 (magenta) and octreotide (lavender) highlighting the probable clash between octreotide D-Trp

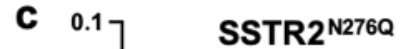

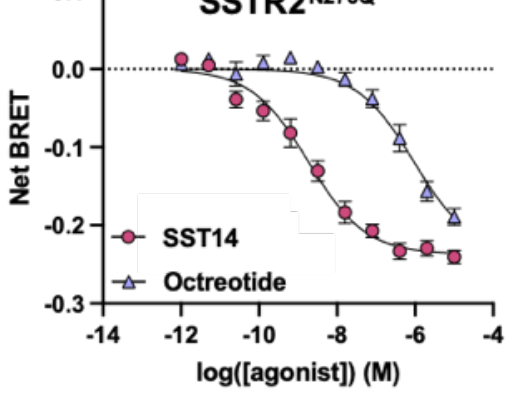

d

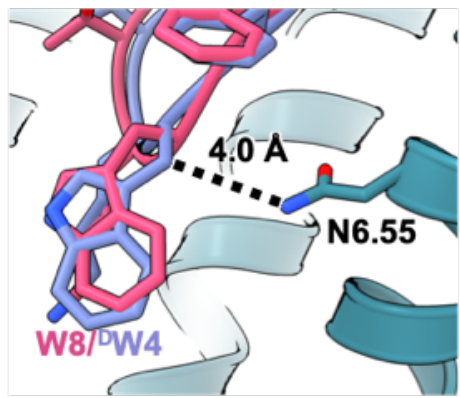

$\log ([$ agonist]) (M)
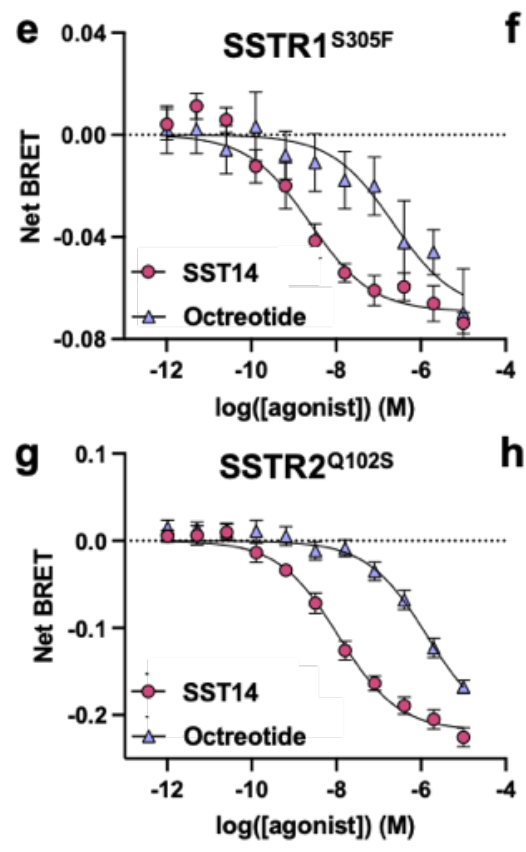

h

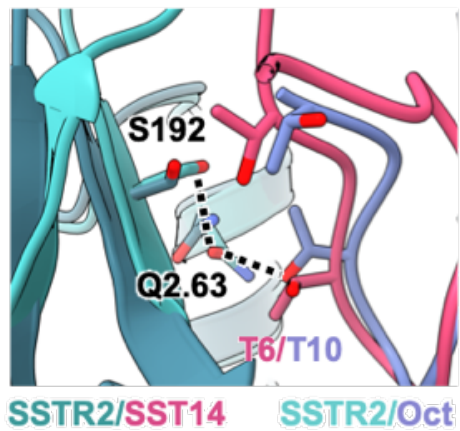
and a N6.55Q mutation as in SSTR1 and SSTR4. e, Dose-response curves for SSTR $1^{\text {S305F }}$-dependent activation of Gi3, $\mathbf{f}$, Overlay of SSTR2 (teal) bound to SST14 (magenta) and octreotide (lavender) highlighting the position of F7.35. g, Doseresponse curves for SSTR2 ${ }^{\text {Q102S }}$. dependent activation of $\mathrm{Gi} 3$, h, Overlay of SSTR2/SST14 structure (dark teal/magenta) with SSTR2/octreotide (light teal/lavender) highlighting differences in hydrogen bonding between T6/T10 of the ligands and ECL2/ Q102 2.63 . Error bars are S.E.M. 
Despite the complexity in ECL-driven receptor subtype selectivity, we were able to identify more straightforward sources of ligand discrimination in the TM bundle between

211 SSTR1/4 and SSTR2/3/5. Prior work has suggested that mutating two SSTR1 TM bundle

212 residues to the analogous residues of SSTR2, S3057.35 F and Q291 ${ }^{6.55} \mathrm{~N}$, can produce

213 near-SSTR2 levels of octreotide binding ${ }^{5}$. Indeed, while SSTR2 but not SSTR1 is

214 activated by octreotide, $\mathrm{N} 276^{6.55} \mathrm{Q}$ in SSTR2 decreases octreotide signaling in a selective

215 manner (Fig. 4c). This is consistent with our structural data, as the $\beta$-carbon of D-Trp in

216 octreotide is only roughly $4 \AA$ away from N276 6.55 (Fig. 4d), clashing with a glutamine

217 residue that would otherwise be accommodated by the L-Trp $\beta$-carbon of SST14.

218 Furthermore, the effects of the $S 305^{7.35} \mathrm{~F}$ mutation in SSTR1 (Fig. 4e) can be rationalized

219 by considering F294 ${ }^{7.35}$ in SSTR2, which lies below F6 in SST14 and the disulfide bond

220 of octreotide (Fig. 4f), thus providing hydrophobic packing. Given the likelihood of

221 significant conformational differences in peptide binding between subtypes, it seems

222 plausible that the much larger phenylalanine of the $S 305^{7.35} \mathrm{~F}$ mutation in SSTR1 would

223 lock octreotide into a binding conformation akin to that of SSTR2. This model is consistent

224 with prior studies that suggest the $\mathrm{Q} 291^{6.55} \mathrm{~N}$ alone does little to improve octreotide

225 binding to SSTR1 ${ }^{5}$. The SSTR2 structures also identify a novel source of SSTR1/4 versus

226 SSTR2/3/5 selectivity. Although conserved between octreotide and SST14, T6/T10

227 (octreotide/SST14) is slightly twisted in octreotide compared to SST14, positioning this

228 residue to interact with Q102 2.63 (Fig. 4h). This glutamine, conserved in SSTR3 and 5 and

229 replaced by a serine in SSTR1 and 4, appears important for ligand selectivity, as the

230 Q102 $2.63 \mathrm{~S}$ mutant in SSTR2 selectively reduces octreotide-induced G protein signaling

231 (Fig. 4g). 


\section{Discussion}

In this study, we have presented structures of active-state SSTR2 in complex with

234 either its native peptide agonist or a clinically used synthetic agonist. In combination with

235 the structure of unliganded, inactive-state SSTR2 we report in an accompanying

236 manuscript ${ }^{12}$, these structural snapshots provide a detailed view of the receptor activation

237 mechanism and the interactions of the key tryptophan-lysine motif of SSTR peptide

238 agonists. Our structural analysis and assays highlight a duality in the nature of subtype

239 selectivity at SSTRs. The basis of selectivity in the TM bundles of SSTR1/4 versus

240 SSTR2/3/5 is straightforward and largely attributable to three residues, yet complicated

241 in the extracellular loops, where chimeric receptors have a non-linear effect on SST14-

242 induced signaling. While it is clear from these studies that some aspects of subtype

243 selectivity are structural, it is likely, given our discovery of the dynamic behavior of ECL2

244 and the observed non-linearity in ligand recognition of ECL chimeras, that some aspect

245 of SSTR subtype selectivity is kinetically driven, as is the case for cannabinoid receptors ${ }^{21}$

246 and adrenergic receptors ${ }^{22}$. However, further structural, kinetic, and dynamics studies will

247 be necessary to fully tease apart the intricacies of ligand recognition in this complex

248 system. Despite this, the combination of high-resolution structures, precise

249 characterization of activation mechanism, and a firm understanding of how residues in

250 the transmembrane helices of SSTRs generate subtype selectivity provides a starting

251 framework for the design of next-generation SSTR agonists.

252

\section{Author Contributions}


254 M.J.R. Cloned constructs, expressed and purified proteins, processed EM data, built

255 models, and ran/analyzed molecular dynamics simulations. J.M. performed BRET

256 assays, expressed and purified proteins. O.P. prepared cryo-EM samples and collected

257 cryo-EM data. K.B. M.J.R. and G.S. wrote the manuscript with input from J.M. and O.P..

258 G.S. supervised the project.

260 Data Availability

261 All data generated or analyzed in this study are included in this article and the 262 Supplementary Information. The cryo-EM density maps and corresponding coordinates

263 have been deposited in the Electron Microscopy Data Bank (EMDB) and the Protein Data 264 Bank (PDB), respectively, under the following accession codes: XXXX YYYY

267 Ethics Declarations

268 Competing Interests

269 The authors declare no competing interests.

\section{Acknowledgements}

272 Cryo-EM data were collected at the Stanford cryo-EM center (cEMc) with support from E.

273 Montabana. This work was supported, in part, by the Mathers Foundation (G.S.), training

274 grant T32GM089626 (J.G.M.) and used the Extreme Science and Engineering Discovery

275 Environment (XSEDE) $)^{23}$ resource comet-gpu through sdsc-comet allocation TG- 
276 MCB190153 (G.S.), which is supported by National Science Foundation grant number

$277 \mathrm{ACl}-1548562$.

279 Methods

\section{Construct generation}

281 SSTR2 was obtained from Horizon Discovery in cDNA and cloned into a pFastBac vector

282 containing an N-terminal haemagglutinin (HA) signal sequence followed by a FLAG

283 epitope (DYKDDDDK), the $29 \mathrm{~N}$-terminal residues (M1 to D29) of the $\beta 2$ adrenergic

284 receptor and TEV protease cleavage site; the $\mathrm{C}$ terminus contained a C3 protease

285 cleavage site followed by enhanced green fluorescent protein (eGFP) and a hexahistine

286 (His6) tag. The cloning was performed with Gibson cloning. The ICL3 and part of TM6

287 from Kappa opioid receptor was inserted as described in Robertson et al. ${ }^{12}$. Mutants and

288 ECL swaps were generated with Q5 site directed mutagenesis.

290 Expression and Purification of SSTR2-GFP

291 Baculovirus expressing SSTR2KappalCL3-GFP at P2 was used to infect Sf9 insect cells

292 (Expression Systems) at a density of 3-4 million cells $/ \mathrm{ml}$ and incubated at $28 \mathrm{C}$ with

293 shaking. After 48 hours, cells were collected with centrifugation at 1500xg, washed with

294 phosphate-buffered saline containing $2 \mu \mathrm{M}$ agonist, and pellets were snap frozen in liquid

295 nitrogen for purification. Pellets containing SSTR2KарраICL3-GFP were resuspended in

296 hypotonic buffer containing $20 \mathrm{mM}$ HEPES $\mathrm{pH}$ 7.5, $5 \mathrm{mM} \mathrm{MgCl}$, protease inhibitor

297 cocktail, benzonase, 2 uM agonist (either octreotide or SST14), and $2 \mathrm{mg} / \mathrm{ml}$

298 iodoacetamide and gently stirred at 4C for an hour. Lysed membranes were harvested by 
centrifugation at 100,000xg. Membranes were resuspended in solubilization buffer containing 100 mM NaCl, 20 mM HEPES pH 7.5, protease inhibitor cocktail, 1 mM MgCl2, 10\% glycerol, 2 uM agonist (either octreotide or SST14), and $2 \mathrm{mg} / \mathrm{ml}$ iodoacetamide and drip frozen in liquid nitrogen. On the day of purification, membranes were rapidly thawed and detergent was added dropwise to a final concentration of $1 \%$ LMNG, $0.2 \%$ CHS, $0.2 \%$ cholate and allowed to solubilize while gently stirring at $4 \mathrm{C}$ for 3 hours. Insoluble debris was removed with ultracentrifugation at 100,000xg, and solubilized receptor was supplemented with $20 \mathrm{mM}$ imidazole and gravity loaded over Ni-NTA resin at $4 \mathrm{C}$. The resin was washed with 10 column volume $(\mathrm{CV})$ of buffer containing $100 \mathrm{mM} \mathrm{NaCl}, 20 \mathrm{mM}$ HEPES pH 7.5, 2 uM agonist, 0.1\% LMNG, 0.01\% CHS, and $20 \mathrm{mM}$ imidazole and protein was eluted in buffer consisting of $100 \mathrm{mM} \mathrm{NaCl}, 20 \mathrm{mM}$ HEPES pH 7.5, 2 uM agonist,

311 supplemented with $5 \mathrm{mM} \mathrm{CaCl}_{2}$ and gravity loaded over $\mathrm{M} 1$ flag resin. The flag resin was

312 washed with $10 \mathrm{CV}$ of $100 \mathrm{mM} \mathrm{NaCl}, 20 \mathrm{mM} \mathrm{HEPES} \mathrm{pH} \mathrm{7.5,} 2$ uM agonist, 0.01\% LMNG,

$3130.001 \% \mathrm{CHS}$, and $2 \mathrm{mM} \mathrm{CaCl}_{2}$. Protein was eluted in $100 \mathrm{mM} \mathrm{NaCl}, 20 \mathrm{mM} \mathrm{HEPES} \mathrm{pH}$ $3147.5,2$ uM agonist, 0.01\% LMNG, 0.001\% CHS, 10\% glycerol, $0.1 \mathrm{mg} / \mathrm{ml}$ FLAG peptide

315 and 2 mM EDTA, prior to concentration and injection onto size exclusion chromatography 316 (SEC) with buffer consisting of $100 \mathrm{mM} \mathrm{NaCl}, 20 \mathrm{mM} \mathrm{HEPES} \mathrm{pH} \mathrm{7.5,} 2$ uM agonist, 0.01\% 317 LMNG, 0.001\% CHS. Monomeric receptor was pooled, supplemented with glycerol, 318 concentrated, and flash frozen in liquid nitrogen for later complexation. 
321 The secreted single chain construct of Fab16 (scFv16) recognizes an epitope composed

322 of the terminal part of the $\alpha \mathrm{N}$ helix of Gai1 as well as part of the G $\beta 1$ subunit ${ }^{13}$ and was

323 generated as previously described ${ }^{15}$. Purified scFv16 was concentrated to $13 \mathrm{mg} / \mathrm{ml}$ and

324 flash frozen in buffer containing 20 mM HEPES pH 7.5, $100 \mathrm{mM} \mathrm{NaCl}$, and 15\% glycerol

325 for later use.

327 Purification of Gi protein heterotrimer

328 Pellets containing DN Gai3 were thawed and resuspended in lysis buffer containing 20 $329 \mathrm{mM}$ HEPES pH 75, 1 mM EDTA pH 8, 5\% glycerol, 1 mM MgCl 2,5 uM $\beta$-mercaptoethanol,

330100 uM GDP, protease inhibitor cocktail, and benzonase. Resuspended pellets were 331 gently stirred for 20 minutes at $4 \mathrm{C}$ before pelleting membranes by ultracentrifugation at

$332100,000 x g$ for 30 min. Membranes were resuspended with douncing in a glass tissue 333 grinder in $100 \mathrm{mM} \mathrm{NaCl}, 20 \mathrm{mM}$ HEPES pH 7.5, 1\% sodium cholate, 5\% glycerol, $1 \mathrm{mM}$

$334 \mathrm{MgCl}_{2}, 5 \mathrm{mM} \quad \beta$-mercaptoethanol, $100 \mathrm{uM}$ GDP, protease inhibitor cocktail, and 335 benzonase. Solubilization further proceeded with 60 minutes of gentle stirring at 4C 336 before insoluble debris was removed by ultracentrifugation at 100,000xg for 50 minutes.

337 Solubilized $\mathrm{G}$ protein was supplemented with $30 \mathrm{mM}$ imidazole and incubated with Ni-

338 NTA beads for 1 hour. Beads were loaded onto a glass gravity column and washed with

$33910 \mathrm{CV}$ with increasing concentrations of LMNG/CHS and decreasing concentrations of 340 cholate until a final wash in $100 \mathrm{mM} \mathrm{NaCl}, 20 \mathrm{mM} \mathrm{HEPES} \mathrm{pH} \mathrm{7.5,} \mathrm{0.05 \%} \mathrm{LMNG,} \mathrm{0.005 \%}$

$341 \mathrm{CHS}, 5 \%$ glycerol, $1 \mathrm{mM} \mathrm{MgCl} 2,5 \mathrm{mM} \beta$-mercaptoethanol, $100 \mathrm{uM}$ GDP, and $30 \mathrm{mM}$

342 imidazole, before being eluted into the same buffer containing $250 \mathrm{mM}$ imidazole. G

343 protein heterotrimer was then supplemented with $1 \mathrm{mg}$ HRV-3C protease/50 mg 
344 heterotrimer to remove the $6 x \mathrm{His}$ tag and incubated overnight at $4 \mathrm{C}$ with dialysis against

345 low imidazole buffer. The following day dialyzed protein was flowed through a Ni-NTA

346 gravity column to remove HRV-3C and uncleaved heterotrimer, concentrated, and

347 injected onto SEC with a buffer containing $100 \mathrm{mM} \mathrm{NaCl}, 20 \mathrm{mM} \mathrm{HEPES} \mathrm{pH} \mathrm{7.5,} \mathrm{0.01 \%}$

348 LMNG, 0.001\% CHS, 1 mM MgCl , 100 uM TCEP, 20 uM GDP, and 5\% glycerol.

349 Fractions containing heterotrimer were pooled, concentrated, and flash frozen in liquid 350 nitrogen for later use.

\section{Formation of SSTR2/Gi3 complex}

353 Aliquots of SSTR2 KappalCL3-GFP and DN Gai3 were thawed and mixed in a buffer

354 containing 100 mM NaCl, 20 mM HEPES pH 7.5, 0.01\% LMNG, 0.001\% CHS, 20 uM

355 TCEP, 0.1mM MnCl2, lambda phosphatase, and 200 uM agonist peptide (either SST14

356 or octreotide), and incubated at room temperature for 1 hour. Complex was then

357 incubated with 3C protease, apyrase, and scFV16 for 3 hours on ice. The mixture was

358 then diluted 10-fold with buffer containing $100 \mathrm{mM} \mathrm{NaCl}, 20 \mathrm{mM}$ HEPES pH 7.5, 0.01\%

359 LMNG, 0.001\% CHS, $10 \mathrm{uM}$ agonist peptide, and $5 \mathrm{mM} \mathrm{CaCl} 2$ and loaded onto $\mathrm{M} 1$ anti-

360 DYKDDDDK immunoaffinity beads. Beads were washed with $10 \mathrm{CV}$ of buffer containing

361100 mM NaCl, 20 mM HEPES pH 7.5, 0.004\% LMNG, 0.0004\% CHS, 10 uM agonist

362 peptide, and $1 \mathrm{mM} \mathrm{CaCl}_{2}$ to remove excess $\mathrm{G}$ protein and scFv before elution with 0.1

$363 \mathrm{mg} / \mathrm{ml}$ FLAG peptide and 2 mM EDTA. Complex was concentrated and injected onto an 364 enrich 650 SEC column equilibrated with buffer containing 100 mM NaCl, 20 mM HEPES

$365 \mathrm{pH} 7.5,0.001 \%$ LMNG, 0.00033\% GDN, 0.0001\% CHS, and $10 \mathrm{uM}$ agonist peptide and 366 fractions were spiked with 200 uM agonist peptide upon elution. Monomeric fractions 
367 were pooled, concentrated to $5-15 \mathrm{mg} / \mathrm{ml}$, and used to freeze grids for cryogenic electron

368 microscopy.

370 Cryo-EM sample preparation and data collection

371 All samples were prepared on glow-discharged holey gold grids (Quantifoil ultrAufoil

372 R1.2/1.3), blotted in an FEI Vitrobot Mark IV (Thermo Fisher Scientific) at 4C and 100\%

373 humidity, and plunge frozen into liquid ethane. Blotting conditions for each sample were

374 as follows: 3.5 ul of SSTR2/SST14/Gi3/scFv16 complex at $15 \mathrm{mg} / \mathrm{ml}$ with an additional

$3750.025 \%$ beta OG, and 3.5 ul of SSTR2/octreotide/Gi3/scFv16 complex at $9 \mathrm{mg} / \mathrm{ml}$.

376 Cryo-EM data were collected with a KRIOS electron microscope at an accelerating

377 voltage of $300 \mathrm{kV}$ using SerialEM with beam tilt compensation on a Gatan K3 direct

378 electron detector. The resulting image stacks have a pixel size of $0.426 \AA$ in super

379 resolution mode. Each SSTR2/Gi3/Octreotide image stack is composed of 55 frames with

380 an incident electron dose of 1.22 e-/Å2 per frame, for a total dose of 67 e-/Å2/s per

381 micrograph. Each SSTR2/Gi3/SST14 image stack is composed of 50 frames with an

382 incident electron dose of 1.04 e-/Å2 per frame, for a total dose of 52 e-/Å2/s per 383 micrograph.

\section{Data Processing}

386 All datasets were initially imported into Relion $3.1^{24}$ for motion correction with 387 MotionCorr2 ${ }^{25}$, CTF estimation with CTFFIND426, and template-based particle picking.

388 Extracted particles were then imported into CryoSPARC ${ }^{27}$ for 2D classification, 3D 389 classification, and initial nonuniform refinement. Cleaned particle stacks were then 
390 transferred back to Relion 3.1 for Bayesian polishing, before being returned to

391 CryoSPARC for final 2D cleaning, non-uniform, and local refinement. A pictorial flowchart

392 of the data processing workflows can be found in Extended Data Figs. 2c, 3c.

\section{Model Building}

395 Ligand placement was performed using a modification of the GemSpot pipeline ${ }^{28}$ to allow 396 for placement of cyclic peptides. A custom library of ring conformations of the major ring 397 was built for each peptide using PrimeMCS sampling ${ }^{29}$. This library was then used with 398 GlideEM to produce a set of initial potential ligand poses. For each of these ligand poses, 399 the full system, including protein, ligand and co-factors, was optimized using Phenix$400 \mathrm{OPLS}^{30}$ to produce the final poses.

401

\section{Molecular Dynamics Simulations}

403 The apo inactive state structure of SSTR2 determined in our accompanying manuscript 404 was used to set up molecular dynamics simulations. Inactive receptor with octreotide was 405 generated by aligning the active octreotide structure and translating the peptide to avoid 406 steric clashes. Both systems were oriented with the OPM webserver ${ }^{31}$ in a lipid bilayer.

407 The oriented systems were solvated in a box of POPC/CHS lipid bilayer, TIP3P water, 408 and $150 \mathrm{mM} \mathrm{NaCl}$ with the CHARMM-GUI ${ }^{32}$. CHARMM36m ${ }^{33}$ input files with hydrogen 409 mass repartitioning were taken from the CHARMM-GUI and used for MD simulations. The 410 NAMD software package ${ }^{34}$ was used to execute simulations employing a Langevin

411 thermostat and a Nosé-Hoover Langevin piston barostat at $1 \mathrm{~atm}$ with a period of 50 fs 412 and decay of 25 fs; periodic boundary conditions were used with nonbonded interactions 
413 smoothed starting at $10 \AA$ to $12 \AA$ with long-range interactions treated with particle mesh

414 Ewald (PME). A 2 fs timestep with SHAKE and SETTLE algorithms ${ }^{35,36}$ was used during

415 equilibration and a 4 fs timestep during production. The system was minimized for 1,500

416 steps, heated from 0 to $303.15 \mathrm{~K}$ in $20 \mathrm{~K}$ increments simulating for $0.4 \mathrm{~ns}$ at each interval,

417 and an additional $10 \mathrm{~ns}$ of equilibration was run at $10 \mathrm{~ns}$; a $1 \mathrm{kcal} / \mathrm{mol} / \AA 22$ harmonic

418 restraint was applied to all non-hydrogen, non-water, and non-ion atoms for each of these

419 steps. This was followed by 10 ns of equilibration with restraints applied to only non-

420 hydrogen protein atoms, and then another 10 ns of equilibration with only CA atom

421 restraints. 30 ns of unrestrained simulation was also considered to be equilibration;

422 production simulations were performed for $1.0 \mu \mathrm{s}$. All simulations were run in triplicate

423 with different initial velocity seeds for each condition. The distance between the center of

424 mass of the receptor and the center of mass of W188 was measured in $\mathrm{VMD}^{37}$.

425

\section{JAWS calculations}

427 JAWS simulation ${ }^{17}$ input files from the SSTR2/Gi3/SST14 complex structure were 428 generated from a pdb file of all atoms within 25 A of the tip of the Gi3 C-terminal helix.

429 The JAWS preparation scripts of the GemSpot pipeline ${ }^{28}$ were used to convert input files.

430 A $15 \AA$ sphere around region of interest was solvated with theta waters to be sampled in

431 the simulation and protein sidechains in this region were treated as flexible. The protein 432 was simulated with the OPLS-AA/M force field ${ }^{38}$, and the TIP4P model ${ }^{39}$ was used for the 433 water, with $\mathrm{MCPRO}^{40}$ used to for the JAWS Monte Carlo simulations. We ran 5 million 434 steps for solvent equilibration, 10 million in hydration site identification, and 50 million in 435 the production phase. Displayed waters are those where there was strong agreement 
436 between triplicate simulations in position and binding energy was estimated to be

437 favorable $(<0 \mathrm{kcal} / \mathrm{mol})$.

\section{BRET-based assays}

440 For BRET-based assays, SST14 (Cayman, 20809) was prepared in citrate buffer pH 4.8,

441 octreotide (Cayman, 23757) in citrate buffer pH 4.8, neurotensin(8-13) (MedChem

442 Express, HY-P0251) in water, and (-)-isoproterenol hydrochloride (Sigma, 16504) in water.

443 All ligands were prepared at $10 \mathrm{mM}$, aliquoted, and stored at $-80 \mathrm{C}$ for later use. Ligands

444 used for cryoEM studies were prepared in water at a concentration of $10 \mathrm{mM}$. G protein

445 BRET assays were performed as previously described (32367019) with the following

446 modifications: HEK-293S cells grown in FreeStyle 293 suspension media (Thermo

447 Fisher) were transfected at a density of 1 million cells/mL in $2 \mathrm{~mL}$ volume using $1200 \mathrm{ng}$

448 total DNA at 1:1:1:1 ratio of receptor: $\mathrm{G}_{\alpha}: \mathrm{G}_{\beta}: \mathrm{G}_{\gamma}$ and a DNA:PEI ratio of $1: 5$, and incubated

449 in a 24 deep well plate at $220 \mathrm{rpm}, 37^{\circ} \mathrm{C}$ for 48 hours. Cells were harvested by

450 centrifugation, washed with Hank's Balanced Salt Solution (HBSS) without

451 Calcium/Magnesium (Gibco), and resuspended in assay buffer (HBSS with $20 \mathrm{mM}$

452 HEPES pH 7.45) with $5 \mu \mathrm{g} / \mathrm{mL}$ freshly prepared coelenterazine 400a (GoldBio). Cells

453 were then placed in white-walled, white-bottom 96 well plates (Costar) in a volume of 60

$454 \mathrm{\mu l} /$ well and 60,000 cells/well. Drug dilutions were prepared in drug buffer (assay buffer

455 with $0.1 \% \mathrm{BSA}, 6 \mathrm{mM} \mathrm{CaCl}, 6 \mathrm{mM} \mathrm{MgCl}_{2}$ ), of which $30 \mu$ were immediately added to

456 plated cells. Ten minutes after the addition of ligand, plates were read using a SpectraMax

457 iD5 plate reader using $585 \mathrm{~nm}$ and $525 \mathrm{~nm}$ emission filters with a one second integration 
458 time per well. The computed BRET ratios (GFP2/RLuc8 emission) were normalized to

459 ligand-free control (Net BRET) prior to further analysis.

460

\section{Cell surface expression testing}

462 Transfection of HEK-293S cells were performed with identical conditions as for BRET 463 assays. Cells were washed with FACS buffer (1\% BSA in PBS), stained with mouse anti464 FLAG primary antibody (Sigma) in FACS buffer, washed three times, stained with Alexa 465 Fluor 647 Goat Anti-Mouse IgG (Abcam), washed an additional three times with FACS 466 buffer, and read on a NovoCyte Quanteon running NovoExpress v1.3.0 (Agilent) with $467>50,000$ cells counted. Total expression levels (arbitrary units) were obtained via the 468 product of percent positive by gating and median level of positive counts, and normalized 469 to wild-type for at least 3 independent biological replicates.

470

471 Statistical analysis

472 Normalized 11-point dose-response curves in technical duplicate were analyzed by 473 simultaneous curve-fitting of at least 3 biological replicates (minimum 66 data 474 points/curve) using a log(dose) vs. response model in Prism 9.1.0 for macOS (GraphPad 475 Software) as previously described ${ }^{10}$. All 95\% confidence intervals for $\mathrm{EC}_{50}$ and $\mathrm{E}_{\max }$ were 476 asymmetrically calculated, and S.E.M. of EC50 ratios were symmetrically calculated. All

477 statistical comparisons between $\mathrm{EC}_{50}$ and $\mathrm{EC}_{50}$ ratios were performed with analysis of 478 variance (ANOVA) (extra sum-of-squares F Test or one-way ANOVA) with correction for 479 multiple hypothesis testing as previously described ${ }^{10}$. 


\section{References}

482 1. Günther, T. et al. International union of basic and clinical pharmacology. CV. somatostatin receptors: Structure, function, ligands, and new nomenclature. Pharmacol. Rev. (2018). doi:10.1124/pr.117.015388

2. Gu, Y. Z. \& Schonbrunn, A. Coupling specificity between somatostatin receptor sst2A and $G$ proteins: Isolation of the receptor-G protein complex with a receptor antibody. Mol. Endocrinol. (1997). doi:10.1210/mend.11.5.9926

3. Hofman, M. S., Eddie Lau, W. F. \& Hicks, R. J. Somatostatin receptor imaging with68Ga DOTATATE PET/CT: Clinical utility, normal patterns, pearls, and pitfalls in interpretation1. Radiographics (2015). doi:10.1148/rg.352140164

491 4. Kaltsas, G., Androulakis, I. I., De Herder, W. W. \& Grossman, A. B. Paraneoplastic syndromes secondary to neuroendocrine tumours. Endocrine-Related Cancer (2010). doi:10.1677/ERC-10-0024

5. Liapakis, G. et al. Identification of ligand binding determinants in the somatostatin receptor subtypes 1 and 2. J. Biol. Chem. (1996). doi:10.1074/jbc.271.34.20331

6. Bruns, C., Lewis, I., Briner, U., Meno-Tetang, G. \& Weckbecker, G. SOM230: A novel somatostatin peptidomimetic with broad somatotropin release inhibiting factor (SRIF) receptor binding and a unique antisecretory profile. Eur. J. Endocrinol.

500 7. Casarini, A. P. M. et al. Acromegaly: Correlation between expression of

503 8. PLÖCKINGER, U., DIENEMANN, D. \& QUABBE, H.-J. Gastrointestinal Side- 
Effects of Octreotide during Long Term Treatment of Acromegaly*. J. Clin. Endocrinol. Metab. (1990). doi:10.1210/jcem-71-6-1658

9. Parry, J. J., Chen, R., Andrews, R., Lears, K. A. \& Rogers, B. E. Identification of critical residues involved in ligand binding and $G$ protein signaling in human somatostatin receptor subtype 2. Endocrinology (2012). doi:10.1210/en.2011-1662

509 10. Olsen, R. H. J. et al. TRUPATH, an open-source biosensor platform for interrogating the GPCR transducerome. Nat. Chem. Biol. (2020). doi:10.1038/s41589-020-0535-8

512 11. Che, T. et al. Nanobody-enabled monitoring of kappa opioid receptor states. Nat. Commun. (2020). doi:10.1038/s41467-020-14889-7

514 12. Robertson, M. J. et al. Structure Determination of Inactive-State GPCRs with a Universal Nanobody. (2021).

516 13. Maeda, S. et al. Development of an antibody fragment that stabilizes GPCR/Gprotein complexes. Nat. Commun. (2018). doi:10.1038/s41467-018-06002-w

14. Maeda, S., Qu, Q., Robertson, M. J., Skiniotis, G. \& Kobilka, B. K. Structures of the

521 15. Koehl, A. et al. Structure of the $\mu$-opioid receptor-Gi protein complex. Nature 558, $547-552$ (2018).

523 16. Ballesteros, J. A. \& Weinstein, H. Integrated methods for the construction of threedimensional models and computational probing of structure-function relations in $\mathrm{G}$ protein-coupled receptors. Methods Neurosci. (1995). doi:10.1016/S10439471(05)80049-7 
527 17. Michel, J., Tirado-Rives, J. \& Jorgensen, W. L. Prediction of the water content in

528 protein binding sites. J. Phys. Chem. B 113, 13337-13346 (2009).

529 18. Wacker, D. et al. Structural features for functional selectivity at serotonin receptors.

$530 \quad$ Science (80-. ). (2013). doi:10.1126/science.1232808

531 19. Deupi, X., Standfuss, J. \& Schertler, G. Conserved activation pathways in G-

532 protein-coupled receptors. Biochemical Society Transactions (2012).

533 doi:10.1042/BST20120001

534 20. McAllister, S. D. et al. Structural Mimicry in Class A G Protein-coupled Receptor

535 Rotamer Toggle Switches. J. Biol. Chem. (2004). doi:10.1074/jbc.m406648200

536 21. Xing, C. et al. Cryo-EM Structure of the Human Cannabinoid Receptor CB2-Gi

537 Signaling Complex. Cell (2020). doi:10.1016/j.cell.2020.01.007

538 22. Xu, X. et al. Binding pathway determines norepinephrine selectivity for the human

$539 \quad$ B1AR over $\beta 2 A R$. Cell Res. (2021). doi:10.1038/s41422-020-00424-2

540 23. Towns, J. et al. XSEDE: Accelerating scientific discovery. Comput. Sci. Eng.

$541 \quad$ (2014). doi:10.1109/MCSE.2014.80

542 24. Zivanov, J. et al. RELION-3: New tools for automated high-resolution cryo-EM 543 structure determination. bioRxiv (2018). doi:10.1101/421123

544 25. Zheng, S. Q. et al. MotionCor2: Anisotropic correction of beam-induced motion for 545 improved cryo-electron microscopy. Nature Methods 14, 331-332 (2017).

546 26. Rohou, A. \& Grigorieff, N. CTFFIND4: Fast and accurate defocus estimation from 547 electron micrographs. J. Struct. Biol. 192, 216-221 (2015).

548 27. Punjani, A., Rubinstein, J. L., Fleet, D. J. \& Brubaker, M. A. CryoSPARC: Algorithms 549 for rapid unsupervised cryo-EM structure determination. Nat. Methods (2017). 
551 28. Robertson, M. J., van Zundert, G. C. P., Borrelli, K. \& Skiniotis, G. GemSpot: A Pipeline for Robust Modeling of Ligands into Cryo-EM Maps. Structure (2020). doi:10.1016/j.str.2020.04.018

554 29. Sindhikara, D. et al. Improving Accuracy, Diversity, and Speed with Prime Macrocycle Conformational Sampling. J. Chem. Inf. Model. (2017). doi:10.1021/acs.jcim.7b00052

557 30. van Zundert, G. C. P., Moriarty, N. W., Sobolev, O. V., Adams, P. D. \& Borrelli, K. W. Macromolecular refinement of X-ray and cryoelectron microscopy structures with Phenix/OPLS3e for improved structure and ligand quality. Structure (2021). doi:10.1016/j.str.2021.03.011

561 31. Lomize, M. A., Pogozheva, I. D., Joo, H., Mosberg, H. I. \& Lomize, A. L. OPM database and PPM web server: Resources for positioning of proteins in membranes. Nucleic Acids Res. 40, (2012).

564 32. Lee, J. et al. CHARMM-GUI Input Generator for NAMD, GROMACS, AMBER, 565 OpenMM, and CHARMM/OpenMM Simulations Using the CHARMM36 Additive Force Field. J. Chem. Theory Comput. 12, 405-413 (2016).

567 33. Huang, J. et al. CHARMM36m: An improved force field for folded and intrinsically disordered proteins. Nat. Methods 14, 71-73 (2016).

569 34. Phillips, J. C. et al. Scalable molecular dynamics with NAMD. Journal of Computational Chemistry 26, 1781-1802 (2005).

571 35. Miyamoto, S. \& Kollman, P. A. Settle: An analytical version of the SHAKE and 572 RATTLE algorithm for rigid water models. J. Comput. Chem. (1992). 
574 36. Ryckaert, J. P., Ciccotti, G. \& Berendsen, H. J. C. Numerical integration of the 575 cartesian equations of motion of a system with constraints: molecular dynamics of 576 n-alkanes. J. Comput. Phys. (1977). doi:10.1016/0021-9991(77)90098-5

577 37. Humphrey, W., Dalke, A. \& Schulten, K. VMD: Visual molecular dynamics. J. Mol. $578 \quad$ Graph. 14, 33-38 (1996).

579 38. Robertson, M. J., Tirado-Rives, J. \& Jorgensen, W. L. Improved Peptide and Protein Torsional Energetics with the OPLS-AA Force Field. J. Chem. Theory Comput. 11,

582 39. Jorgensen, W. L., Chandrasekhar, J., Madura, J. D., Impey, R. W. \& Klein, M. L. 583 Comparison of simple potential functions for simulating liquid water. J. Chem. Phys. 79, 926-935 (1983).

585 40. Jorgensen, W. L. \& Tirado-Rives, J. Molecular modeling of organic and biomolecular systems using BOSS and MCPRO. J. Comput. Chem. 26, 1689-1700 (2005). 